\title{
CONCEPTION DES PROJETS DE PASSES, SUIVI DES CHANTIERS, COÛTS, ETUDES SUR MODĖLES RÉDUITS
}

\author{
J.P. PORCHER ${ }^{\star}$ et M. LARINIER ${ }^{\star \star}$
}

\begin{abstract}
* Conseil Supérieur de la Pêche - DR 2 - 84, Rue de Rennes - 35510 CESSON SEVIGNE
** CSP-CEMAGREF, GHAAPPE - Institut de Mécanique des Fluides - Avenue du Professeur Camille Soula - 31400 TOULOUSE
\end{abstract}

\section{LA CONCEPTION D'UN PROJET DE PASSE}

\subsection{Recueil d'informations préliminaires}

Une passe à poissons doit répondre à deux impératifs d'égale importance :

- être adaptée à l'espèce (ou aux espèces) auxquelles elle est destinée,

- être adaptée au site sur lequel elle est installée.

La conception d'un ouvrage de franchissement commencera donc par la collecte d'informations biologiques, hydrologiques, topographiques et hydrauliques. Ces éléments devront figurer dans tous les dossiers de passes, leur connaissance étant indispensable pour formuler un avis lors d'une consultation.

\subsubsection{Données biologiques}

On précisera tout d'abord les espèces migratrices qui devront être prises en compte :

- pour les obstacles existants, en consultant notamment les décrets et les arrêtés d'application qui fixent les espèces migratrices à prendre en compte sur les tronçons de cours d'eau classés au titre de l'article L.232-6 du Code Rural,

- pour les renouvellements ou modifications d'autorisations et de concessions ainsi que pour les ouvrages nouveaux, la nécessité de préserver le patrimoine aquatique peut entraîner des exigences plus étendues que celles requises par l'article L.232-6. On sera alors amené, à la demande de l'administration, à prendre en compte les besoins de franchissement d'autres espèces migratrices (holobiotiques et amphibiotiques) présentes, en portant attention à l'existence éventuelle de programmes de restauration de populations de migrateurs sur certains tronçons de cours d'eau.

Dans tous les cas, la liste des espèces migratrices devra être fixée sans ambiguïté avant l'élaboration du projet.

Le dossier technique devra préciser la liste des espèces, et pour chacune d'entre elles, les données disponibles sur les effectifs actuels ou potentiels, les périodes de migration de montaison et de dévalaison, ainsi que les modes d'obtention de ces différentes informations (données bibliographiques, enquêtes, observations de terrain, stations de contrôle situées en aval, etc...).

\subsubsection{Données hydrologiques et qualité des eaux}

Le dossier devra inclure les caractéristiques hydrologiques du cours d'eau, en se référant à la station de jaugeage la plus proche: module interannuel, débits moyens mensuels, débits caractéristiques d'étiage et de crue (DCN10 et DCX10). II précisera 
également l'amplitude de variation des débits en période de migration et indiquera, le cas échéant, comment ces débits sont influencés par des aménagements situés en amont.

Sur des cours d'eau de faible importance, on ne disposera qu'exceptionnellement des résultats d'une station de jaugeage implantée à proximité de l'aménagement. II conviendra alors de rechercher des sites de référence, de préférence sur le même bassin hydrographique, à défaut sur un bassin voisin de nature géologique comparable, à partir duquel des corrélations de débits satisfaisantes pourront être établies. II ne s'agira pas, en général, d'obtenir des informations d'une grande précision, mais de définir les ordres de grandeur des valeurs caractéristiques du débit ainsi que l'allure et l'amplitude de ses variations en période de migration. En dehors de certaines singularités liées aux écoulements de type karstique, les corrélations directes avec les superficies des bassins versants seront souvent suffisantes.

On recherchera également les données concernant la qualité des eaux sur le site :

- la température de l'eau, par ses conséquences sur les capacités de nage et de saut des migrateurs, est susceptible d'influencer notablement l'efficacité d'une passe migratoire, notamment dans les cours d'eau de montagne, soumis à des basses températures. A l'opposé, les températures estivales sur la partie basse des grands cours d'eau peuvent atteindre des valeurs limitantes à certaines périodes pour les salmonidés migrateurs,

- la qualité physico-chimique de l'eau (notamment présence de substances polluantes, teneur en oxygène) est à prendre en compte pour l'alimentation de la passe à poissons et le débit d'appoint nécessaire à son fonctionnement. On portera une attention particulière aux prises d'eau dans les retenues où une stratification thermique ou physicochimique peut se produire : suivant la profondeur à laquelle l'eau est prélevée, on peut rencontrer des températures inadéquates ou des teneurs en oxygène insuffisantes à certaines périodes de l'année.

\subsubsection{Caractéristiques de l'aménagement}

a. Plans de l'aménagement

II est nécessaire de disposer :

- d'un relevé à une échelle qui permette de situer l'ensemble des aménagements du site : prises d'eau, canaux d'amenée et de fuite, organes de restitution, etc...,

- d'un relevé plus précis de la zone (ou des zones) où les migrateurs se trouvent bloqués (montaison et dévalaison),

- d'une coupe des ouvrages aux endroits où le dispositif de franchissement est susceptible d'être installé,

- d'un relevé détaillé des dimensions et cotes de tous les ouvrages de régulation hydrauliques (déversoirs, vannes, prises d'eau et restitutions) ainsi que des caractéristiques des turbines.

On pourra utiliser des plans existants, mais la vérification de la conformité des ouvrages lors d'une visite sur le site est indispensable.

\section{b. Relation entre le débit et les niveaux d'eau amont et aval}

Le dossier technique doit comprendre impérativement la relation débit-cotes des plans d'eau amont et aval.

Un ouvrage de franchissement est en effet calé par rapport au niveau des plans d'eau amont (alimentation de l'ouvrage) et aval (configuration et attractivité de l'entrée pour le poisson). Les niveaux en période d'étiage - qui correspondent généralement au dénivelé maximum - devront figurer explicitement sur les plans. On notera également les cotes des plans d'eau relevées à des débits caractéristiques qui serviront de points de repère dans la conception et la vérification de la plage de fonctionnement du dispositif de franchissement. 
Dans certains cas, on sera amené à installer sur le site des échelles limnimétriques, voire un limnigraphe ou une sonde de niveau. Dans le cas d'ouvrages nouveaux, il est souvent difficile de déterminer avec précision le niveau aval après aménagement qui dépendra à la fois de l'exécution des travaux et de l'évolution ultérieure du lit du cours d'eau. II sera alors recommandé dans la mesure du possible de prévoir une marge de sécurité dans le calage de la partie aval de l'ouvrage.

Dans certains cas (ouvrages fixes déversants), il est possible de déterminer par le calcul de façon assez précise les variations du niveau amont à partir du débit du cours d'eau par l'utilisation des relations classiques charge amont-débit au-dessus de déversoirs.

c. Etude des usages de l'eau sur le site et des modes de fonctionnement des installations hydrauliques

Le dossier technique devra inclure une description précise du mode de fonctionnement des installations et analyser les conséquences des différents cas de figure sur la configuration des écoulements ainsi que leur effet probable sur la migration du poisson, tant à la montée qu'à la descente (attraction des poissons au pied des turbines ou des ouvrages évacuateurs par exemple). On examinera éventuellement l'impact causé par la pratique de chômages et d'éclusées. On apportera un soin particulier à l'étude des problèmes posés par les restitutions multiples (dérivations, turbines, vannes de décharge...). Le cas échéant, des aménagements particuliers du site ou des règles de fonctionnement seront envisagées pour limiter les perturbations ou les blocages de la migration.

d. Recherche des lieux de rassemblement et de tentative de franchissement des migrateurs

Dans le cas d'ouvrages existants sur des cours d'eau fréquentés par les migrateurs, une enquête auprès des services de la pêche, des riverains et des pêcheurs peut permettre de circonscrire les zones de blocage des migrateurs et de disposer ainsi de garanties supplémentaires pour le choix de l'implantation de la passe à poissons. Sur des aménagements complexes et sur les cours d'eau importants, une étude biologique préalable peut s'imposer (observation directe ou enregistrements vidéo sur le site, radiopistage, etc...).

\subsection{Conception de l'ouvrage}

Le travail de conception de l'ouvrage est alimenté par les données listées ci-dessus. On peut en décrire chronologiquement les différentes phases comme suit :

\subsubsection{Détermination de la plage de fonctionnement de la passe à poissons en débits et niveaux}

A partir des données hydrologiques recueillies et des rythmes migratoires, on fixera la gamme de débits du cours d'eau dans laquelle l'ouvrage de franchissement doit rester fonctionnel. II est d'autant plus nécessaire de minimiser les temps de blocage à l'aval des obstacles que la fenêtre d'activité migratoire de l'espèce concernée est étroite et que l'on se trouve dans une période proche de la reproduction (cas des espèces potamotoques). Par exemple,il est indispensable qu'un ouvrage de franchissement destiné à l'alose ou à l'anguille soit opérationnel de façon permanente pendant toute la période de la migration. On pourra en revanche tolérer qu'une passe à poissons destinée aux salmonidés migrateurs soit suralimentée et non fonctionnelle lors de certains pics de crue.

On essaye généralement de faire en sorte que la passe fonctionne correctement pour les débits du cours d'eau compris entre la valeur d'étiage et une valeur voisine de deux à trois fois le module interannuel. Cette dernière valeur correspond pour la plupart des cours d'eau français à un débit qui n'est dépassé qu'une trentaine de jours par an en année moyenne, et seulement quelques jours consécutifs. Cet ordre de grandeur assure en général un fonctionnement permanent de l'ouvrage de franchissement en période de migration de l'alose et une fréquence de fonctionnement satisfaisante pour les salmonidés. 
La plage de débits du cours d'eau dans laquelle la passe doit rester fonctionnelle étant déterminée, on relèvera les niveaux des plans d'eau amont et aval correspondants. Une connaissance précise de ces niveaux est en effet indispensable au calage de l'ouvrage et à son dimensionnement.

\subsubsection{Choix des débits dans la passe à poissons}

On déterminera les débits à utiliser dans le dispositif de franchissement pour attirer et assurer le transit des migrateurs, tant à la montée qu'à la descente. II conviendra de se référer à la réglementation de l'aménagement qui fixe souvent un débit réservé, utilisable en totalité ou en partie pour le fonctionnement des dispositifs de franchissement. On rappelle que le débit dans le dispositif doit être à l'échelle de ceux transitant dans le cours d'eau et l'aménagement.

\subsubsection{Choix du type de passe.}

Dans de nombreux cas, la décision finale résultera d'un compromis, plusieurs types d'ouvrages ou plusieurs configurations pouvant convenir, avec pour chacune d'entre elles des avantages et des inconvénients.

Les principaux facteurs intervenant dans la détermination du type de passe sont rappelés de façon très succincte dans la suite:

- les espèces concernées. Certaines passes sont très spécifiques. C'est notamment le cas des passes à civelles et à anguillettes. De même, les passes à ralentisseurs sont pratiquement réservées aux salmonidés,

- les débits à transiter dans l'ouvrage. Ces débits, lorqu'ils sont faibles (quelques dizaines de $\mathrm{l} / \mathrm{s}$ ) ou au contraire très importants (plusieurs $\mathrm{m}^{3} / \mathrm{s}$ ) deviennent incompatibles avec certains types de dispositifs,

- les variations des niveaux amont et aval. Les différents types d'ouvrages sont plus ou moins sensibles aux variations des plans d'eau amont et aval,

- les contraintes topographiques. Le choix d'un type de passe peut être imposé par des contraintes topographiques sur un site exigu. Une passe à ralentisseurs doit être constituée de parties rectilignes, les changements de direction ne pouvant avoir lieu qu'au niveau de bassins de repos. Ce type d'ouvrage requiert donc un développement conséquent en longueur. La passe à bassins est davantage plastique, les bassins pouvant être repliés et agencés pour s'adapter au terrain. L'ascenseur est en général assez facilement intégrable aux ouvrages hydrauliques : il n'exige qu'une faible emprise au sol,

- la dénivellation à franchir. C'est un facteur important, intervenant dans le coût de l'ouvrage de franchissement, à considérer dans le choix du type de passe : une passe à ralentisseurs peu onéreuse sur un barrage de faible hauteur deviendra moins intéressante qu'une passe à bassins sur un ouvrage plus élevé (nécessité d'installer des bassins de repos). De même, sur un barrage de grande hauteur, l'ascenseur sera une solution moins coûteuse que la passe à bassins,

- le coût de fonctionnement et d'entretien du dispositif de franchissement, qui peut représenter un budget important à long terme. Il constitue un critère à ne pas négliger dans le choix du type d'aménagement. On sera ainsi amené à privilégier les dispositifs statiques (passes à bassins) par rapport aux ouvrages comportant de nombreux organes mobiles (écluses et ascenseurs). On attachera une attention toute particulière aux problèmes de colmatage des prises d'eau et des grilles qui entraînent des interventions fréquentes et coûteuses, et on choisira un type d'ouvrage, une configuration et une implantation propres à faciliter la maintenance,

- le transport solide dans le cours d'eau. Dans le cas d'un transport solide important (galets et blocs), on évitera les passes à ralentisseurs ou les passes dont les bassins trop profonds risqueraient de s'engraver,

- les conditions climatiques, en particulier le gel qui peut affecter le fonctionnement des organes mobiles ou de grilles trop fines. Ce facteur doit être pris en compte notamment pour la conception d'ascenseurs dans des sites de montagne. 


\subsubsection{Implantation sur le site}

L'implantation de la passe à poissons doit tenir compte :

- de l'espace disponible pour l'emprise au sol,

- de la courantologie sur le site dans toute la gamme de débits retenue pour le fonctionnement de la passe (éviter les situations où l'écoulement à la sortie de la passe est masqué ou perturbé),

- des informations collectées sur les rassemblements de migrateurs et les tentatives de franchissement sur les ouvrages existants. On recherchera l'implantation correspondant à la meilleure attractivité de l'entrée pour le poisson. Si la situation la plus adéquate ne peut être retenue, il sera nécessaire de renforcer l'attractivité du dispositif par un débit plus important,

- des problèmes d'entretien.

\subsubsection{Dimensionnement et calage du dispositif}

En se référant aux règles de dimensionnement et/ou aux abaques de calcul donnés pour chaque type de passe, on définira l'ouvrage dans ses moindres détails. Le dossier comprendra les éléments suivants :

- plan de situation du dispositif et de ses annexes,

- plans détaillés comportant profils en long et coupes,

- note descriptive donnant les caractéristiques de la passe : dimensions des bassins, mode de communication entre bassins (dimensions des échancrures, fentes ou orifices), caractéristiques des ralentisseurs, pente...,

- note précisant le comportement hydraulique de la passe pour plusieurs configurations caractéristiques des niveaux amont et aval considérés en période de migration,

- mode de prise, de dissipation et d'injection du débit d'attrait dans l'ouvrage,

- débits et vitesses à l'entrée de la passe en fonction des débits du cours d'eau, dispositifs de protection contre les corps dérivants.

\section{LE SUIVI DE CHANTIER}

\subsection{Généralités}

La maîtrise d'oeuvre pour les chantiers de grosses passes à poissons est équivalente à celle de tout ouvrage d'art de même importance et doit faire appel aux mêmes moyens : elle ne sera pas détaillée ici et on se limitera à signaler la nécessité de contrôler la qualité des matériaux, la fiabilité des matériels hydromécaniques, l'ancrage aux ouvrages existants, de vérifier certaines cotes ou niveaux essentiels (radiers, pertuis d'alimentation ...).

On s'intéressera dans la suite aux équipements de moindre importance, qui peuvent être réalisés par des entreprises non spécialisées et nécessitent de ce fait une vigilance particulière.

\subsection{Programmation du chantier}

Le choix de la date des travaux devra reposer sur une estimation de leur durée prévisible, et retenir une période présentant la garantie de débits modérés. On cherchera de plus à s'assurer que l'entreprise de gros oeuvre présente la disponibilité nécessaire et des moyens matériels suffisants pour réaliser les travaux dans les délais prévus.

Une attention particulière doit être apportée aux problèmes de batardage et de mise hors d'eau du chantier. L'expérience montre que les tentatives d'économie sur ce poste se soldent généralement par un surcroît de dépenses hors de mesure ou par une réalisation non conforme de l'ouvrage. II conviendra donc d'estimer avec précision les besoins de mise hors d'eau et de protection du chantier, les difficultés d'accès ou de prélèvement de matériaux sur place et de mettre en oeuvre tous les moyens nécessaires pour le bon déroulement des travaux. 
Des garanties particulières peuvent être demandées aux entrepreneurs, telles que la prise en compte des risques de crues par une assurance ou l'intervention de pénalités de retard pour la totalité ou certaines phases du chantier. Les contraintes spécifiques (précision des cotes dans certaines parties de l'ouvrage : bassins pour piégeage, échancrures) seront précisées dans le marché et les mécanismes de régulation devront être détaillés dans un cahier des charges.

\subsection{Exécution des travaux}

\subsubsection{Interprétation des plans et implantation de l'ouvrage}

Les plans de l'ouvrage doivent être bien détaillés (proscrire tout projet qui ne reposerait pas sur un levé topographique exact du site, ou pire, les documents du type "schéma établi sur le terrain"). Les cotes au millimètre près sont conseillées et doivent être rattachées à une référence bien définie.

On travaillera sur des plans datés et, au moment de l'installation du chantier, on vérifiera que l'entrepreneur est bien en possession du dernier projet (et de celui-là seulement !). Les plans seront commentés avec le responsable de l'entreprise et on s'assurera qu'il sait qui contacter en cas de problème d'interprétation ou si une modification est imposée par une contrainte de site. Toute modification, aussi minime soit-elle, du projet initial doit être validée par le concepteur du dispositif de franchissement ou par une personne disposant des mêmes compétences.

L'emprise au sol de l'ouvrage sera déterminée avec précision (repères) lors de l'installation du chantier.

\subsubsection{Suivi des travaux}

Des visites périodiques par une personne familière du fonctionnement et des critères de conception des passes à poissons devront être programmées aux stades les plus importants du chantier. On peut recommander notamment les étapes suivantes :

— fin des démolitions ou des fouilles,

- mise en place du ferraillage des fondations et des radiers,

- fin de coffrage, avant mise en oeuvre du béton de gros oeuvre,

- décoffrage et travaux de finition.

A chaque étape, on vérifiera les niveaux des radiers et des seuils, surtout dans la partie aval où on constate une propension à la surélévation des ouvrages pour limiter les démolitions. II faudra contrôler particulièrement l'existence d'une fosse d'appel suffisante au pied de l'ouvrage, les entrepreneurs ayant tendance à réduire, voire combler les fosses à l'aval de l'ouvrage par crainte d'affouillement.

Les finitions de l'ouvrage doivent éviter aux migrateurs de se blesser, notamment au niveau des raccordements aux ouvrages existants, des arêtes qui devront être arrondies ou chanfreinées et des organes métalliques. Les bavettes en caoutchouc pourront être utilisées pour interdire aux migrateurs l'accès de passages sans issue ou de zones où ils sont susceptibles de se blesser.

\subsection{Réception des travaux}

La réception de l'ouvrage se fera de préférence hors d'eau pour procéder à une vérification générale des niveaux de génie civil, à moins que cette vérification n'ait été faite lors des suivis périodiques de chantier. Elle incluera une mise en eau de l'aménagement et une vérification de son fonctionnement hydraulique (mesure des lignes d'eau et calcul des débits transités). On réglera ou on ajustera, le cas échéant, le niveau de certaines échancrures ou déversoirs au moyen de planchettes. Dans ce cas, le réglage définitif sera suivi d'une fixation inamovible des planchettes qui seront de préférence réalisées en béton. 


\section{COÛT DES PASSES A POISSONS}

II est souvent intéressant de déterminer très tôt l'ordre de grandeur du coût d'un dispositif de franchissement.

\subsection{Passes à bassins successifs}

Le coût d'une passe à bassins successifs est approximativement proportionnel au volume interne du génie civil, c'est-à-dire au volume représenté par le produit de la largeur intérieure, la longueur et la profondeur moyenne de la structure de la passe.

Des statistiques récentes (décembre 1992) portant sur une centaine de passes à bassins montrent que le coût moyen (coût H.T.) est d'environ $3600 \mathrm{~F} / \mathrm{m}^{3}$ (médiane 3300 $\mathrm{F} / \mathrm{m}^{3}$, premier et troisième quartile $2300 \mathrm{~F} / \mathrm{m}^{3}$ et $4400 \mathrm{~F} / \mathrm{m}^{3}$ ). La variabilité de ce coût unitaire est cependant assez grande : il peut varier de moins de $2000 \mathrm{~F} / \mathrm{m}^{3}$ à plus de $6000 \mathrm{~F} / \mathrm{m}^{3}$ pour certains ouvrages.

La grande majorité des coûts rentrant dans l'étude statistique sont afférents à des dispositifs de franchissements construits sur des obstacles existants. Les coûts unitaires les plus faibles observés sont relatifs aux passes construites en même temps que l'usine ou le barrage.

Le volume interne du génie civil défini pour estimer le coût d'un ouvrage comprend:

- le volume de tous les bassins, c'est-à-dire de la passe proprement dite,

- le volume nécessaire à la prise, au transit et à la dissipation du débit complémentaire d'attrait,

- le volume nécessaire au piégeage et/ou à la visualisation du poisson.

Seul le volume "utile" des bassins (volume d'eau dans les bassins en étiage) est très tôt et facilement prévisible sur un aménagement donné : il est proportionnel à la chute maximale au niveau de l'obstacle (observée généralement en étiage) et au débit transitant dans la passe. Ce volume est déterminé de telle sorte que la puissance dissipée volumique ne dépasse pas 150 à 200 watts $/ \mathrm{m}^{3}$.

Il convient cependant d'y ajouter :

- un volume correspondant à la prise en compte des fluctuations du niveau d'eau de part et d'autre de l'ouvrage en période de migration (d'une importance très variable suivant les cours d'eau et les obstacles),

- un volume correspondant à la revanche destinée à éviter le débordement de la passe,

- un volume supplémentaire, qui peut être aussi très variable, imposé par la topographie du site et le tracé de la passe.

Il est aussi possible d'estimer le coût en fonction de la chute maximale $(H)$ et du débit (Q) transitant dans l'ouvrage :

$$
\mathrm{C}=\mathrm{KHQ}
$$

où $\mathrm{C}$ : coût de la passe en $F(H . T$.

$H$ : chute maximale (en $m$ ) en étiage

$Q$ : débit $\left(\mathrm{m}^{3} / \mathrm{s}\right)$ dans la passe en étiage

La valeur moyenne du coefficient $\mathrm{K}$ est de $490000 \mathrm{~F}$ par $\mathrm{m}$ de chute et par $\mathrm{m}^{3} / \mathrm{s} \mathrm{de}$ débit. II est susceptible de varier de $150000 \mathrm{~F}$ à plus de $1000000 \mathrm{~F}$ suivant les conditions d'installation de la passe.

L'estimation des coûts à l'aide de l'expression précédente est évidemment beaucoup plus grossière que celle obtenue à partie du volume intérieur du génie civil.

\subsection{Passes à ralentisseurs}

Des statistiques portant sur une cinquantaine de passes à ralentisseurs donnent un coût unitaire moyen de $10600 \mathrm{~F} / \mathrm{m}^{3}$. (médiane $9700 \mathrm{~F} / \mathrm{m}^{3}$, premier et troisième 
quartile $7600 \mathrm{~F} / \mathrm{m}^{3}$ et $12800 \mathrm{~F} / \mathrm{m}^{3}$ ). Comme pour les passes à bassins, la variabilité est cependant assez grande et tient le plus souvent aux conditions spécifiques d'implantation de certains ouvrages.

Comme pour les passes à bassins, il est aussi possible d'estimer le coût en fonction de la chute maximale $(H)$ et du débit $(Q)$ transitant dans l'ouvrage :

$$
\mathrm{C}=\mathrm{KHQ}
$$

La valeur moyenne du coefficient $K$ est de $270000 \mathrm{~F}$ par $\mathrm{m}$ de chute et par $\mathrm{m}^{3 / \mathrm{s}}$ de débit. Il est susceptible de varier de $100000 \mathrm{~F}$ à plus de $800000 \mathrm{~F}$ suivant les ouvrages.

\subsection{Ascenseurs}

Le coût des ascenseurs à truites varie de $100000 \mathrm{~F}$ à $400000 \mathrm{~F}$, celui des ascenseurs à saumons de $300000 \mathrm{~F}$ à $800000 \mathrm{~F}$. Pour la partie mécanique des gros ascenseurs à aloses à système de concentration mécanisé, les coûts sont de l'ordre de $1500000 \mathrm{~F}$ à $2500000 \mathrm{~F}$.

Il convient d'ajouter à ces coûts celui du génie civil nécessaire à l'implantation de l'ascenseur dans l'ouvrage, en particulier des ouvrages de prise, de dissipation et d'injection du débit d'attrait dans le dispositif qui peuvent être d'importances très variables d'un aménagement à l'autre.

\section{LES MODĖLES RÉDUITS PHYSIQUES}

Les essais sur modèle réduit sont fréquemment utilisés lors de la conception des dispositifs de franchissement dont les écoulements sont trop complexes pour pouvoir être approchés de façon analytique.

Les études sur modèles sont généralement destinées :

- à visualiser et à optimiser l'écoulement dans la passe proprement dite pour s'attacher à ce que vitesses, chutes, niveau de turbulence restent compatibles avec les capacités de nage des espèces migratrices concernées et cela pour toutes les conditions de niveaux amont et aval rencontrées de part et d'autre de l'obstacle en période de migration,

- à dimensionner les dispositifs de prise et de restitution des débits complémentaires d'attrait ainsi que la protection de l'ouvrage contre les corps dérivants,

- à résoudre les problèmes liés à l'implantation de la passe, en particulier pour déterminer la position optimale de son entrée en fonction des conditions hydrodynamiques au pied de l'obstacle.

La majorité des types de passes (passes à fentes verticales, passe de type Ice Harbor, passes à ralentisseurs...) ont été mises au point grâce à des études systématiques sur modèle réduit.

Les échelles des modèles utilisés varient le plus souvent de $1 / 3$ à $1 / 10$ pour les modèles de passes proprement dits, à 1/10 - 1/25 pour les modèles destinés à optimiser l'implantation de l'ouvrage sur l'obstacle.

Les lois de similitude applicables aux écoulements dans les passes sont celles de Froude, puisqu'il s'agit généralement d'écoulements à surface libre et à forte turbulence (forces de viscosité négligeables par rapport à celles dues à la turbulence).

Les études sur modèle, bien que coûteuses, sont indispensables pour l'implantation des dispositifs de franchissement sur les gros ouvrages. Elles permettent d'éviter certaines erreurs qu'il serait difficile et très coûteux de rectifier par la suite. 